\title{
The S100B alarmin is a dual-function chaperone suppressing A $\beta$ oligomerization through combined zinc chelation and inhibition of protein aggregation
}

\author{
Joana S. Cristóvão ${ }^{1,2}$, António J. Figueira ${ }^{1,2}$, Ana P. Carapeto ${ }^{1,3}$, Mário S. Rodrigues ${ }^{1,3}$, \\ Isabel Cardoso ${ }^{4,5}$, Cláudio M. Gomes ${ }^{1,2^{*}}$
${ }^{1}$ Biosystems \& Integrative Sciences Institute, Faculdade de Ciências, Universidade de Lisboa, Lisboa, Portugal
${ }^{2}$ Departamento de Química e Bioquímica, Faculdade de Ciências, Universidade de Lisboa, Lisboa, Portugal
${ }^{3}$ Departamento de Física, Faculdade de Ciências, Universidade de Lisboa, Lisboa, Portugal
${ }^{4}$ i3S - Instituto de Investigação e Inovação em Saúde, Universidade do Porto, Porto, Portugal
${ }^{5}$ IBMC - Instituto de Biologia Molecular e Celular, Universidade do Porto, Porto, Portugal \\ * Corresponding author: C.M. Gomes (cmgomes@ @c.ul.pt)
}

ACS Chemical Neuroscience

\section{Supporting Information}
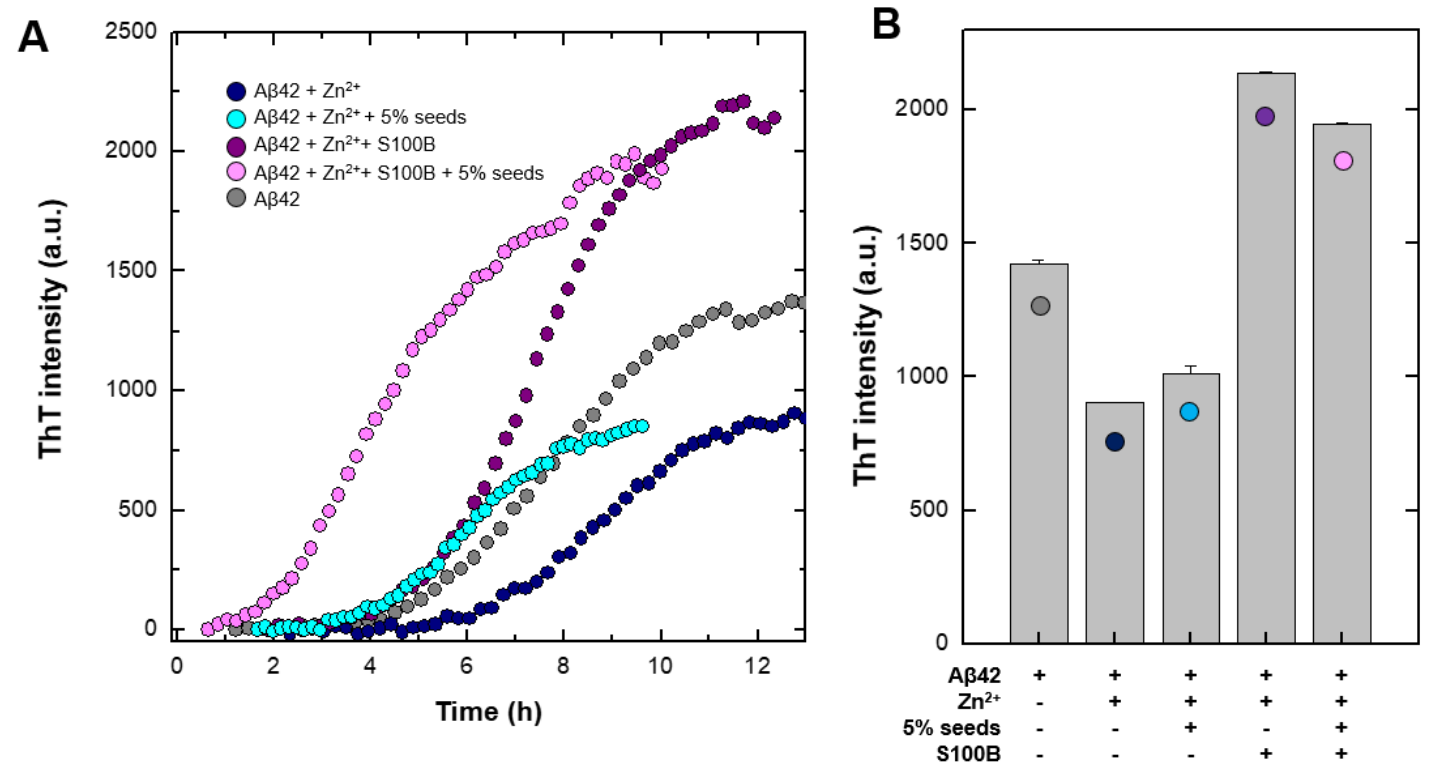

Figure S1 - Effect of S100B over seeded aggregation of $\mathrm{A} \beta 42$ in the presence of $\mathrm{Zn}^{2+}$. A. kinetic traces of ThT-monitored aggregation of A $\beta 42(2 \mu \mathrm{M}$, gray) in the presence of equimolar $\mathrm{Zn}^{2+}$ (dark blue) and with 5\% seeds (light blue), equimolar $\mathrm{Zn}^{2+}$ and S100B (violet) and with 5\% seeds (pink) Averaged traces, $n=3$. B. ThT-intensity at the end point of kinetic traces in different conditions $(n=3)$. 